\title{
Defects \& Remedies in Terry Towel Processing
}

\author{
Ankita Patale, Tushar A Shinde* and Raichurkar PP \\ SVKM's NMIMS, Centre for Textile Functions, India
}

*Corresponding author: Tushar A Shinde, SVKMS, NMIMS, Centre for Textile Functions, Mukesh Patel School of Technology, Management Shirpur, District Dhule-425405, India

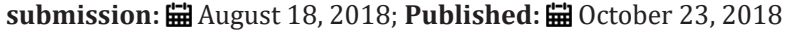

\begin{abstract}
Textile manufacturing is a complex process and it is affected by various parameters. Most of the defect in the fabric is either highlighted or covered in especially in dyeing department. We are follow in this experiment is to identify the important objectionable defect in the terry towel produced in the winch Thies dyeing machine. This research work is done for preventing the defect in processing \& weaving department (excluding spinning department). This study aims to report a root cause analysis of the major defect. This study helps in root cause analysis of the entire process in reducing the defect. It has been reported by many others that careful consideration of process parameter helps technical person to ensure the same faults do not occurs.

This study reviles important process parameters such as pretreatment and its effect on absorbency to make uniform dyeing. This study tries to found the terry towel dyeing defect such as uneven dyeing, staining, lintting, shearing, bowing and holes and how we can minimize and neglect this faults to taking remedies. This report contains work done by various workers and technician in dyeing. Experiment conducted in "Thies" dyeing machine clearly shows how to reduce major objectionable defect in terry towel dyeing. This report contains the important content of parameters and their effect in the reducing the defect significantly. This project is able to give a deep concept about terry towel dyeing faults in soft flow dyeing machine, causes and their remedies and carried out to minimize the dyeing defect by taking particular corrective action Thus, this study enrich knowledge on dyeing, produce faults free terry towel in modern textile plant.
\end{abstract}

Keywords: Quality; Defects; Productivity; Remedies; Dyeing faults

\section{Introduction}

If product fulfills the customer expectation, the customer will be pleased and consider that the product is acceptable and of high quality. If their expectation is not fulfilled, then customer will consider that the product is of low quality (down grade).

Product must be of high quality as much as enough to meet customer requirements. When quality cost is investigated, defect cost is great disadvantages for product cost [1]. The aim of this project is to minimizing the dyeing defect. If these are minimized it is possible to decrease along with increase in production speed. Most of the time is spend in dyeing department for this reason. The dyeing department is most important one concerning production quality study. The defects which are occurred in dyeing department due to various reason, the causes of these defects are determined by defect study. When the causes of these defects are eliminated, the production with better quality (A grade) is achieved.

\section{Material and Method}

\section{Material}

We are used the material for the experiment was $100 \%$ cotton terry towel, which is already prepared from weaving department (excluding for spinning department). So, we are basically focused on the causes \& remedies of following department [2].
a) Weaving
b) Grey Folding
c) Chemical Processing
d) Finish folding

\section{Method}

a) We collected previous data about defect arises in dyeing section.

b) We analyzed data for causes of defect found [3].

c) We took corrective action to minimize the defect by taking precautions in experimental methods.

d) Then collected the data again and analyzed for defect.

e) Trials taken for 25 day and compare the defect by using following unit.

$$
\begin{aligned}
& D H U \%=\frac{\text { Total no. of defect }}{\text { Total pieces checked }} * 100 \\
& \text { Percent defective }=\frac{\text { Defective piece }}{\text { Total pieces checked }} * 100
\end{aligned}
$$

\section{Categorization of defect}

Sewing defect: These defects are usually caused by errors arising from wrong functioning of sewing machine. 
Seaming defect: These defects are usually caused by errors arising from the interaction of the operator and machine in the handling of terry towel.

Fabric defect: These are caused by errors from the fabric processing like pretreatment and dyeing.

Defective pieces: Defective pieces are those pieces, which contain above defects and are separated for alteration during checking.

\section{List of defects (Table 1)}

\section{Table 1:}

\begin{tabular}{|c|c|c|}
\hline $\begin{array}{c}\text { Sr. } \\
\text { No }\end{array}$ & Defect Origin & Defect Name \\
\hline 1 & Weaving & Missing end (terry), Crack, Half pieces, short pieces, Reverse terry, Pile pulling, Lining \\
\hline 2 & Grey Folding & Joint pieces, Yellow marking \\
\hline 3 & Chemical processing & Stain, Shearing, Lintting, Bowing, Patchy, Holes. \\
\hline 4 & Finish folding & Length cut, Cross cut, Selvedge cut, Cross stich over terry, Length stich over terry, Length one side cut, Border cut. \\
\hline
\end{tabular}

\section{Classification of defect}

Defects are classified in three types.
A. Man related defect
B. Machine related defect
C. Material related defect

A. Man related defect: The man related defects mean due to improper handling of materials the defect occurs are like stains, yellow pen marking, colors stains, patchy dyeing, length and cross cut, selvedge cut etc.

B. Machine related defect: The machine related defects are like shearing, bowing, missing end, pile pulling, cracks, half pieces, reverse terry, lining, etc.

C. Material related defect: The material related defect is like contamination, lintting, etc.

\section{Causes of defect and their remedies}

Defect in Dyeing department [4].

Stain: Stains are classified in five types.
a) Soil or mud stain
b) Rust and bit stain
c) Oil stain
d) Contamination stain
e) Softener stain
f) Color stain

\section{a) Soil or mud stains}

Causes Soil or mud stains:

\section{Measurement of defect}

Quantitative measure of defect there are two measuring units as below.

a) Defect per hundred units

b) Percent defectives

$D H U \%=\frac{\text { Total } \text { no. of defect }}{\text { Total pieces checked }} * 100$

Percent defective $=\frac{\text { Defective piece }}{\text { Total pieces checked }} * 100$ i. Improper material handling by workers.

ii. Material in contact with dust present part of machine, trolley, etc.

Remedies Soil or mud stains:

i. Care must be taken during material handling by workers.

ii. To avoid material in contact with dusty part of machine, trolley etc.

\section{b) Rust and bit stains (Figure 1)}

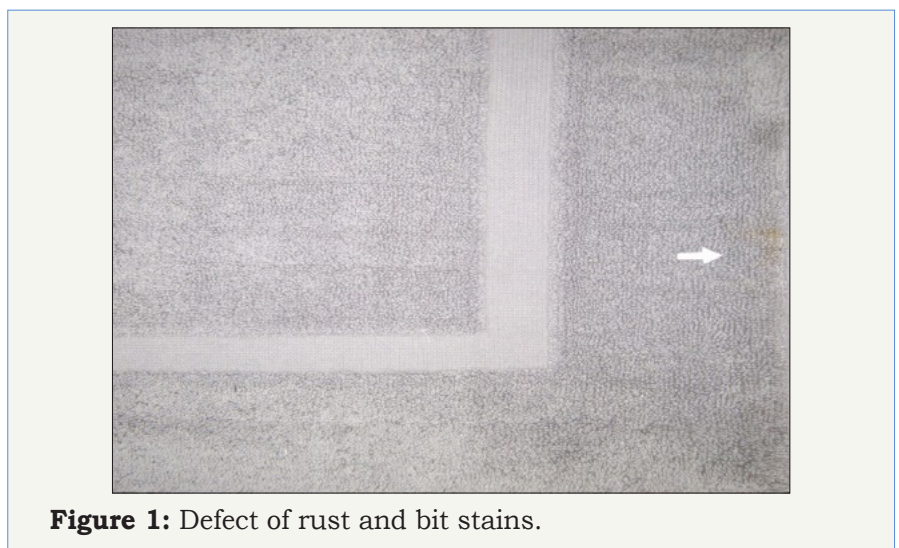

Causes rust and bit stains:

i. Unloading of dyed material in without cover trolley (uncover) and transferring it to next process.

ii. This is occurring when material is in contact with rust present roller on rope opener, shearing machine and trolleys.

iii. Bit stain occurs due to sparrows present in the plant (Figure 2A \& 2B).

Remedies rust and bit stains: 


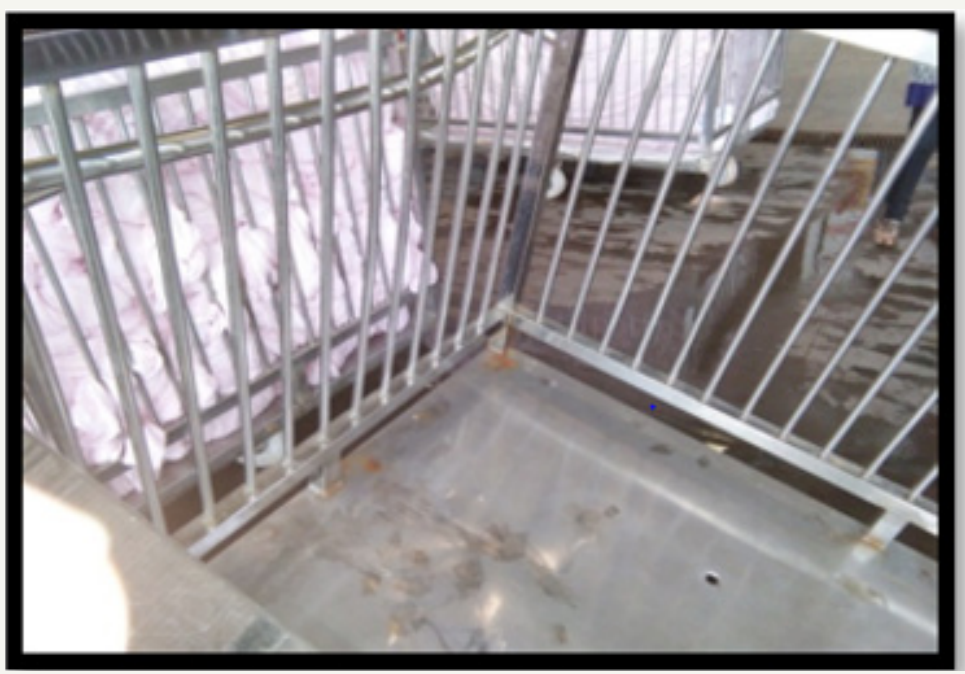

Figure 2A: Causes of rust and bit stains.

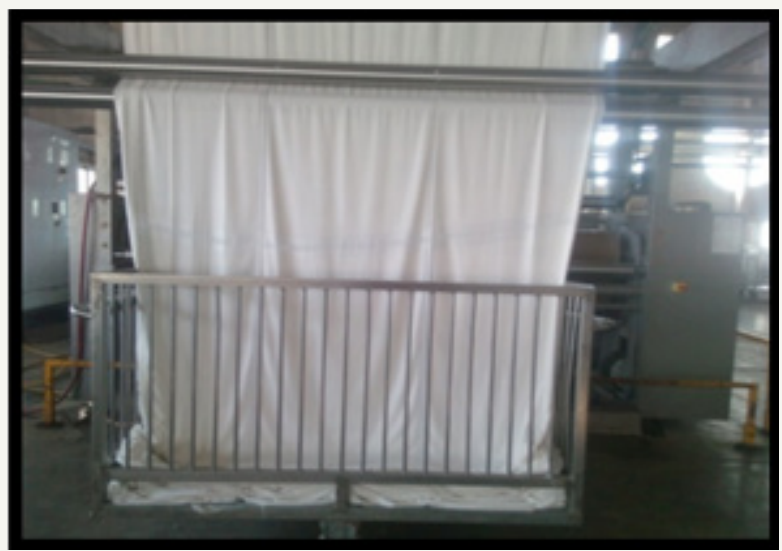

Figure 2B: Causes of rust and bit stains.

i. Loading or unloading the material in full polypropylene sheet covered trolley and then transfer to next process. ii. Proper cleaning and washing of rope opener and shearing machine and trolleys etc.

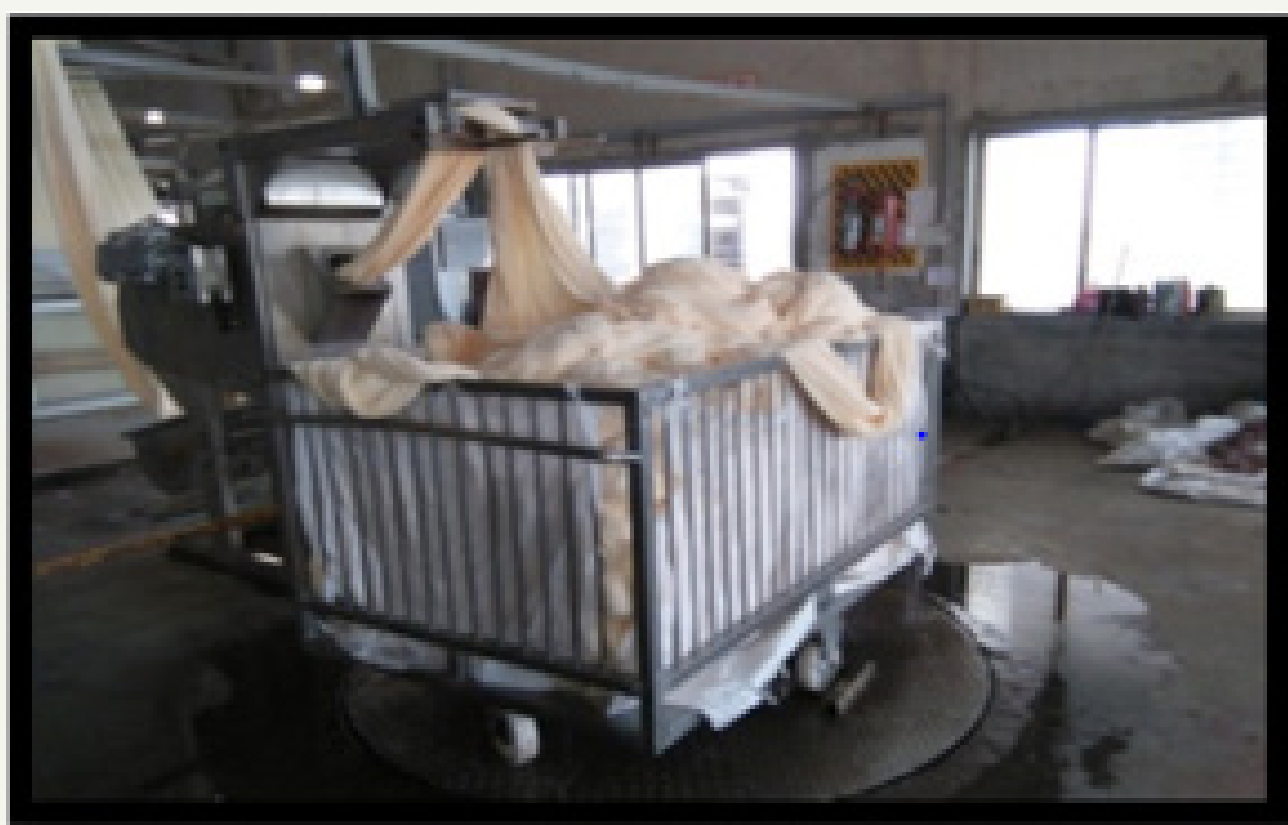

Figure 3A: remedies of rust and bit stains. 
iii. Material must be properly covered with polythene before transfer to next process (Figure 3A \& 3B).

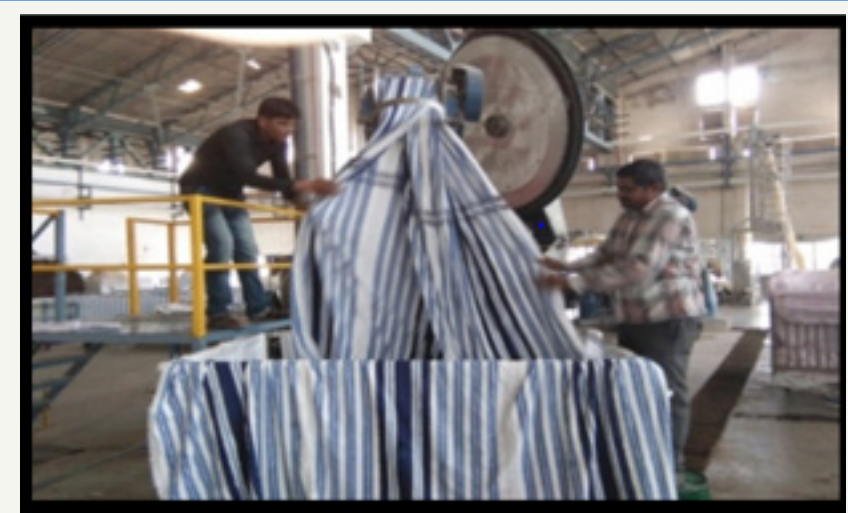

Figure 3B: Causes of rust and bit stains.

\section{c) Oil stain}

Causes of oil stain:

i. Oil stains may be occurring from weaving on selvedge of terry towel due to temple and cutter.

ii. Due to use of excessive oil in dyeing section.

iii. Excessive oil use in stitching machine also causes to oil mark on terry towel (Figure 4).

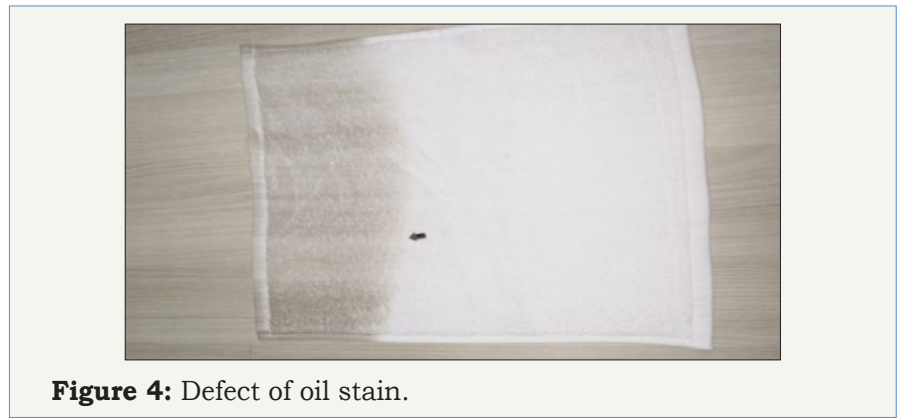

Remedies of oil stain:

i. Daily checking the part of weaving machine and properly clean the machine.

ii. Material should be handle carefully from oily part of machine, trolley etc.

iii. Material should be handle carefully by workers on stitching machine and machine must be clean properly.

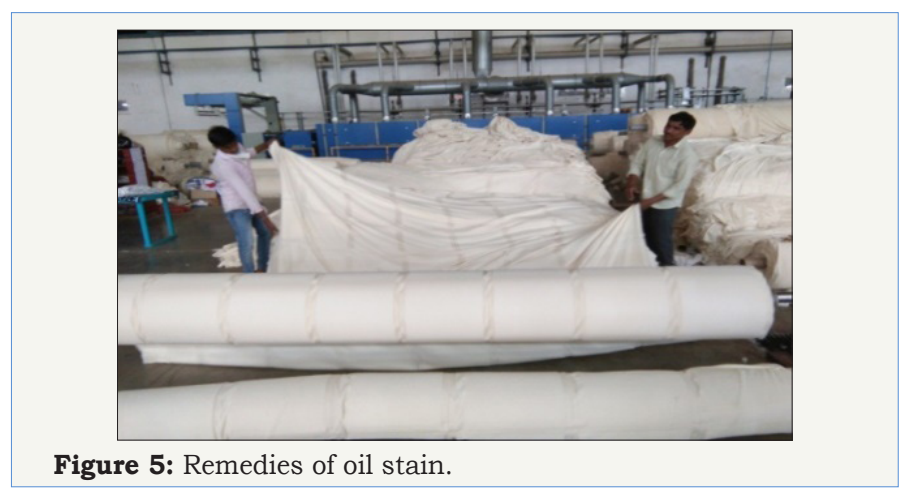

iv. Check grey Material coming from weaving before pretreatment (Figure 5).

\section{d) Contamination}

Causes of Contamination:

i. This stain occurs due to impurities present in row cotton material and it remains as it is up to yarn stage.

Remedies of Contamination:

i. Use impurity less row material of cotton.

\section{e) Softener spot (Figure 6)}

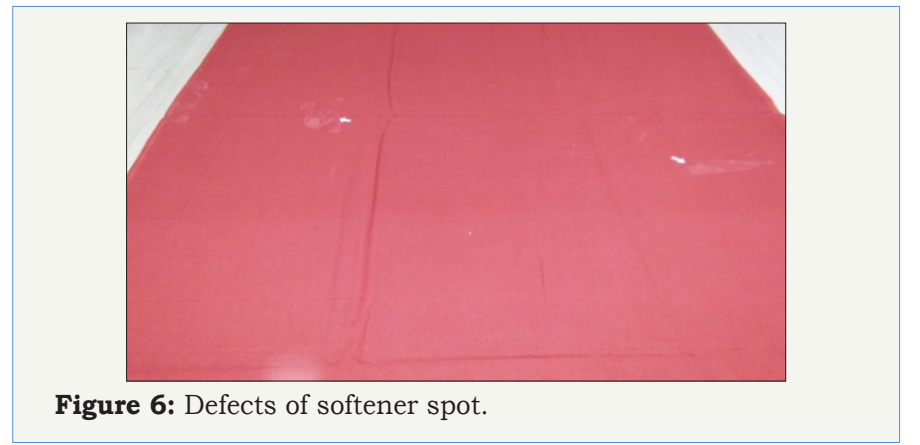

Causes of softener spot:

i. Improper softener dissolving in the addition tank with water.

ii. Improper dosing and poor emulsification of softener applied.

iii. Foam present on material may causes softener spot.

iv. Inferior quality of softener.

v. If $\mathrm{pH}$ is not maintaining.

Remedies of softener spot:

i. Proper or uniformly dissolving of softener in addition tank with water

ii. Proper dosing of softener.

iii. Foam completely removes from fabric.

iv. Use right softener and the correct procedure of application.

v. Maintain $\mathrm{pH}$.

f) Color spot or stain (Figure 7)

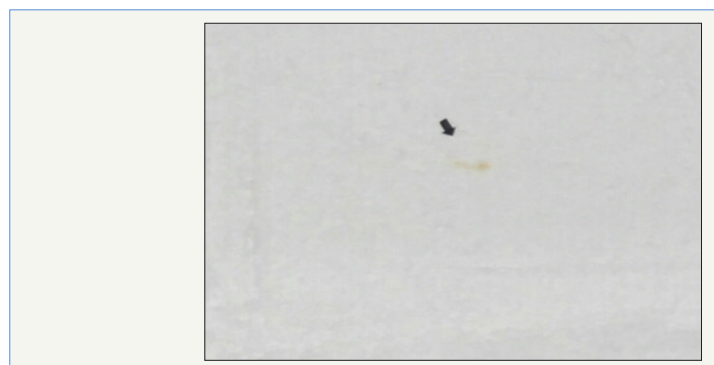

Figure 7: Defects of color Spot. 
Causes of color spot:

i. Improper machine cleaning (hydro extractor, rope opener)

ii. Improper material handling by workers.

iii. Improper mixing and dissolving of dyestuff.

iv. Dye bath hardness.
Remedies of color spot:

i. Proper machine cleaning.

ii. Proper material handling by worker with hand gloze.

iii. Mixing and dosing of dyestuff should be properly.

iv. Water of dye bath should hardness free (Figure $8 \mathrm{~A} \& 8 \mathrm{~B}$ ).
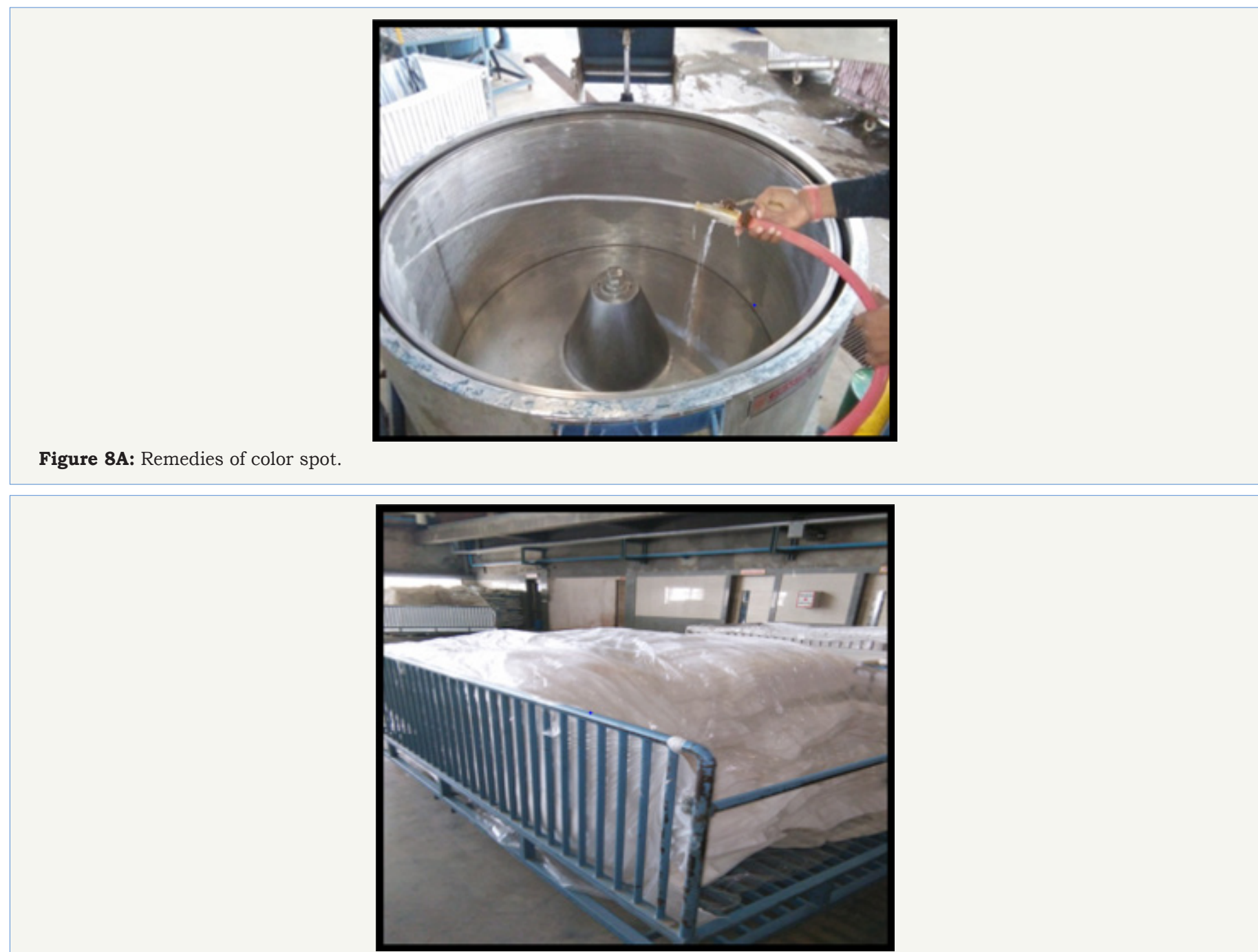

Figure 8B: Remedies of color spot.

\section{Dyeing hole defect (Figure 9):}

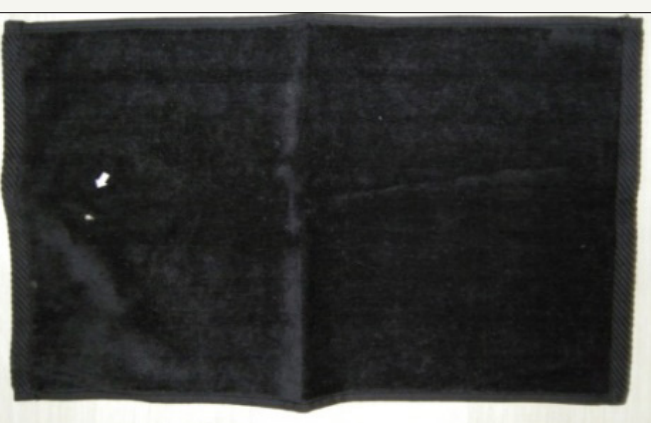

Figure 9: Defect of dyeing hole.
Causes of dyeing hole:

i. Improper scouring and bleaching.

ii. Improper peroxide and caustic dosing.

iii. Due to over bleach.

iv. Improper material handling may causes hole on the fabric. Remedies of dyeing hole:

i. Enzyme should be done carefully.

ii. Caustic dosing must be slow.

iii. Avoided over bleach of material. 


\section{Linttin defect (Figure 10):}

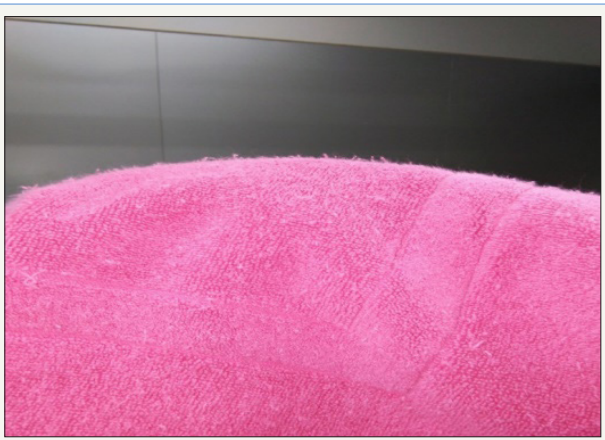

Figure 10: Linttin defect.

Causes of Linttin defect:

i. Improper sizing.

ii. Also due to use of low twist yarn in terry weaving

Remedies of Linttin defect: i. Only minor floats can be rectified.

\section{Defect of bowing (Figure 11):}

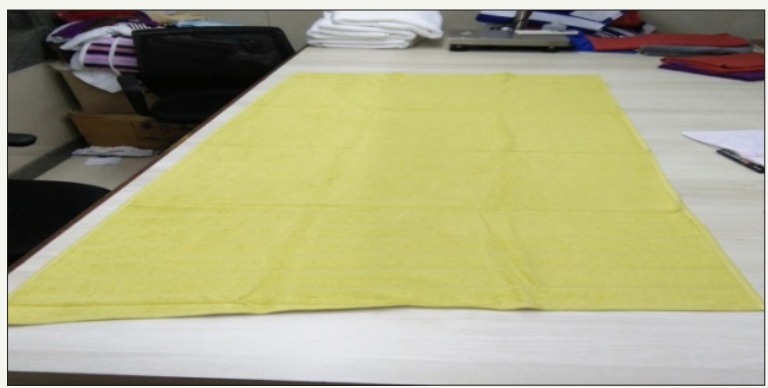

Figure 11: Defect of bowing

Causes of bowing defects:

i. Improper speed of dryer Cylinder.

Remedies of bowing defects:

i. Set proper speed for particular GMS of terry towel.

\section{Shearing (Figure 12):}

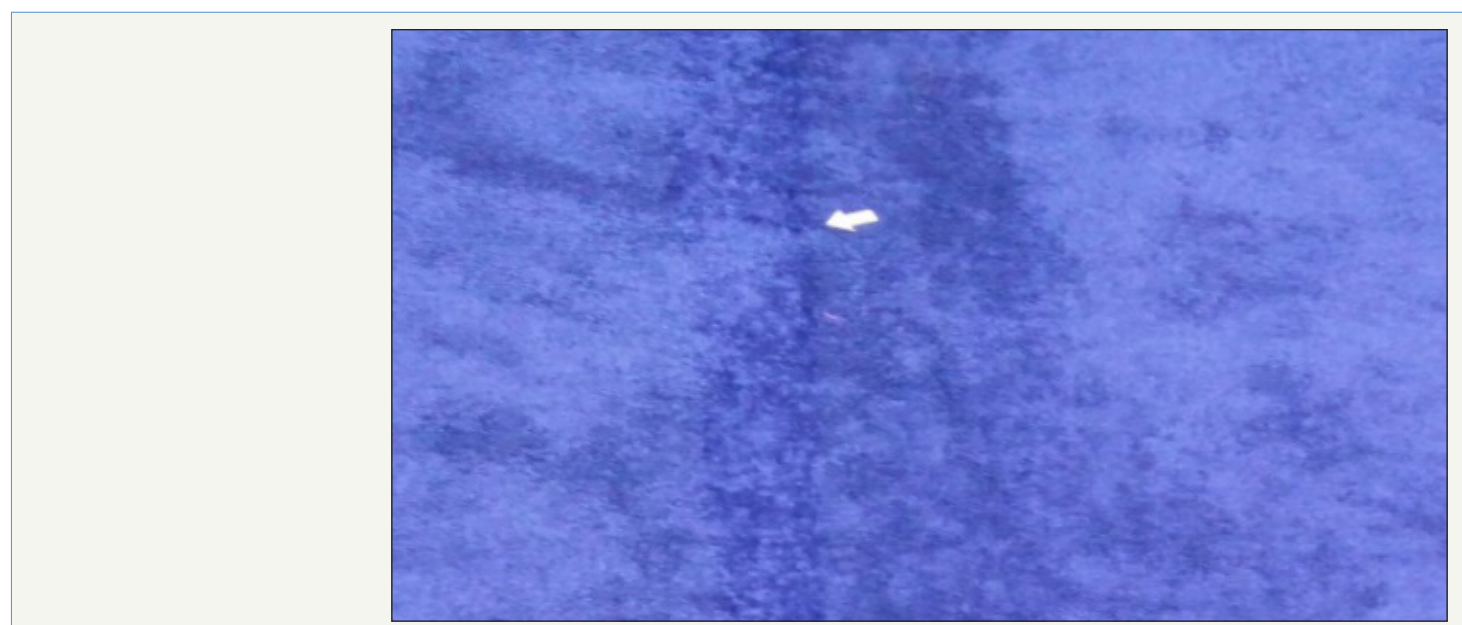

Figure 12: Defect of shearing

Causes of Shearing:

i. Improper distance between spiral blade of shearing machine and material.

ii. Mechanical problem. iii. Operator problem.

Remedies of Shearing:

i. Set proper distance between spiral blade and material.

ii. Operator should be trained and must be careful.

\section{Uneven dyeing (Patchy) (Figure 13):}


Causes of uneven dyeing (Patchy):

i. Uneven pretreatment (desizing, scouring and bleaching).

ii. Very rapid addition of dyes and chemicals.

iii. Lack of controlling dyeing parameters.

Remedies of uneven dyeing (Patchy): i. By ensuring pretreatment.

ii. Proper addition of dyes and chemicals.

iii. Controlling all dyeing parameters.

Process control parameters to avoided uneven dyeing: $\mathrm{pH}$, Time, Temperature, Liquor ratio, Water hardness, Chemical dosing time, H2O2 killing.

Factors and benefits (Table 2)

Table 2:

\begin{tabular}{|c|c|c|}
\hline Sr. No & Factors & Benefits \\
\hline 1 & Minimization of defect & Increase production with A grade \\
\hline 2 & Improve production quality & Acrease cost of terry towel \\
\hline 3 & Checking of grey material & Aefect before dyeing \\
\hline
\end{tabular}

\section{Experimental Work}

\section{Before trial (Figure 14)}

\begin{tabular}{|c|c|c|c|c|c|c|c|c|c|c|c|c|c|c|c|c|c|c|c|c|c|c|c|c|c|c|c|c|c|}
\hline \multirow{3}{*}{\multicolumn{2}{|c|}{\begin{tabular}{|l|l|} 
& $\begin{array}{l}\text { Defect } \\
\text { origin }\end{array}$ \\
\end{tabular}}} & \multicolumn{28}{|c|}{ BEFORE TRALL DATA COULCTION } \\
\hline & & \multicolumn{28}{|c|}{ Dans } \\
\hline & & $\begin{array}{l}\text { Name of } \\
\text { Defest }\end{array}$ & 1 & 2 & 3 & 4 & 5 & 6 & 7 & 8 & , & 10 & 11 & \begin{tabular}{|l|}
12 \\
\end{tabular} & 13 & 14 & 15 & 16 & 17 & is & 19 & 20 & 21 & 22 & 23 & 24 & 25 & \begin{tabular}{|l|} 
Total \\
PYK
\end{tabular} & $\%$ \\
\hline \multirow{8}{*}{ : } & \multirow{8}{*}{$\begin{array}{l}\text { Processi } \\
\text { vs }\end{array}$} & stain & & 11 & $\begin{array}{l}28 \\
4\end{array}$ & & & 59 & \begin{tabular}{|l|}
21 \\
3
\end{tabular} & 49 & & 6 & 8 & & & 59 & & 14 & 31 & 48 & 23 & & 6 & 1 & 1 & & & 23 & 19.79 \\
\hline & & Shearing & & 4 & 4 & & & & 4 & & & 9 & 4 & & & & & 24 & 52 & 34 & & & & 39 & 16 & & & 150 & 45 \\
\hline & & Lintting & & 3 & 3 & & & & & & & 26 & & & & & & 6 & 15 & $\mathrm{zu}$ & & & & 3 & 6 & & & 8 & 199 \\
\hline & & Bowing & & & 1 & & & & 6 & & & 2 & 2 & & & 2 & 4 & & & & 4 & & & & & & & a & 0.5 \\
\hline & & Patchy & & & & & & & & & & & & & & & & & 13 & & & & & & & & & 13 & 03 \\
\hline & & Smail hoie & 3 & 2 & 37 & 16 & 23 & 8 & 31 & 10 & 37 & 6 & 2 & \begin{tabular}{|l|l|}
3 \\
\end{tabular} & 8 & 12 & 1 & 2 & 11 & 3 & 16 & 7 & 2 & 6 & 3 & 4 & 32 & 226 & 728 \\
\hline & & Bizhoie & & & & & 1 & & & & & & & & & & & & & & & & & & & 26 & & 27 & 0.6 \\
\hline & & Total & & & & & & & & & & & & & & & & & & & & & & & & & & 1457 & 0.745 \\
\hline \multirow[b]{2}{*}{2} & \multirow[b]{2}{*}{$\begin{array}{l}\text { Grey } \\
\text { Folding }\end{array}$} & Joint pieces & & 1 & 18 & & & 2 & 7 & 2 & & & 22 & & & & as & & is & 2 & 1 & & 1 & 1 & 12 & & & 112 & 269 \\
\hline & & $\begin{array}{l}\text { Grey } \\
\text { morking }\end{array}$ & & 3 & 25 & & & 5 & 4 & 5 & & & 2 & & & 4 & 2 & 1 & 5 & & & & & 1 & & & & 57 & 1.37 \\
\hline & & Total & & & & & & & & & & & & & & & & & & & & & & & & & & 169 & 0.08 \\
\hline \multirow{9}{*}{3} & \multirow{8}{*}{$\begin{array}{l}\text { Finish } \\
\text { Foiding }\end{array}$} & Length out & & & 15 & & & 7 & 4 & 3 & & 7 & 6 & 10 & & 14 & 15 & , & 20 & 6 & 3 & & \begin{tabular}{|l|}
12 \\
\end{tabular} & 20 & 16 & & & \begin{tabular}{|l|}
167 \\
\end{tabular} & 4.01 \\
\hline & & Cross ant & & 1 & 18 & & & 3 & 34 & 11 & 83 & 1 & , & \begin{tabular}{|l|}
10 \\
3
\end{tabular} & & 16 & 6 & 4 & \begin{tabular}{|l|}
18 \\
\end{tabular} & is & 19 & 9 & 5 & 3 & 18 & & 17 & 402 & 9.64 \\
\hline & & $\begin{array}{l}\text { Selvedige } \\
\text { at }\end{array}$ & ? & & & 60 & & & & & 29 & & 2 & & & & & & & & 67 & 6 & & 3 & 4 & 30 & 151 & $3 n$ & 9.18 \\
\hline & & $\begin{array}{l}\text { Cross stion } \\
\text { or }\end{array}$ & & 3 & 53 & & & 24 & 54 & 12 & & 9 & 25 & & & 30 & 10 & 3 & $2 a$ & 14 & , & & & 6 & 13 & & & 250 & 6.97 \\
\hline & & $\begin{array}{l}\text { length stion } \\
\text { of }\end{array}$ & & 3 & 32 & & & 2 & is & 5 & 1 & 11 & 4 & $a$ & & 16 & 5 & 1 & 24 & 16 & 4 & & 6 & 4 & 7 & & & 205 & 5.02 \\
\hline & & $\begin{array}{l}\text { length ane } \\
\text { sise ast }\end{array}$ & & & & 4 & & & & & 4 & & & 4 & & & & & & & Is & 27 & & & & 20 & 112 & 28 & 6.2 \\
\hline & & $\begin{array}{l}\text { Moie } \\
\text { stioning }\end{array}$ & & & & & & & & & & & & & & & & & & & 2 & & & & & & & 2 & 0.94 \\
\hline & & Barder at & & 3 & & & & & & & & & & & & & & & & & 1 & & & & & & & 4 & 0.09 \\
\hline & \multirow{8}{*}{ Weaving } & Total & & & & & & & & & & & & & & & & & & & & & & & & & & 1714 & 0.8 \\
\hline \multirow{7}{*}{4} & & Missing end & & 10 & 40 & & & 6 & 33 & 22 & & 4 & 1 & & & 17 & \begin{tabular}{|l|}
16 \\
\end{tabular} & 13 & \begin{tabular}{|l|}
51 \\
\end{tabular} & 29 & 2 & & 2 & 4 & 1 & & & 231 & 6.03 \\
\hline & & Pile puling & & 2 & 23 & & & 4 & 6 & 13 & & & 1 & & & & & 4 & 24 & 9 & 1 & & 1 & 1 & 4 & & & 93 & 2.23 \\
\hline & & crood & & 6 & $\omega$ & & & 10 & 27 & \begin{tabular}{|l|}
14 \\
\end{tabular} & & 4 & 3 & & & 12 & 6 & 29 & 76 & 19 & 10 & & 1 & 10 & 5 & & & 120 & 7.69 \\
\hline & & Kail pieces & & & & & 56 & & & & & & & $\$ 4$ & 37 & & & & & & & & & & & & & \begin{tabular}{|l|}
167 \\
\end{tabular} & \begin{tabular}{|l|l|}
353 \\
\end{tabular} \\
\hline & & Short pieces & & & & & & & & & & & & & & & & & & & 6 & & & & & & & 6 & 0.14 \\
\hline & & Stwane To"y & & & & & & & & & & & & & & & & & & 2 & & & & & & & & 2 & 0.04 \\
\hline & & $\sin$ & & & & & & & & & & & & & & & & & & & & & & & & & & & \\
\hline & & Fout & & & & & & & & & & & & & & & & & & & & & & & & & & \begin{tabular}{|l|l|} 
\\
\end{tabular} & 0.4 \\
\hline & & Fout & is & $n$ & 61: & 20 & 40 & 12 & 43 & 246 & เม่ & is & 42 & 242 & 4 & 202 & 20 & 326 & 36 & 224 & 206 & 4 & 36 & 202 & 506 & 20 & in2 & A:s & 212 \\
\hline & & 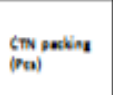 & 7 & $m 2$ & $3_{3}^{m 3}$ & $\begin{array}{l}n: a \\
2\end{array}$ & $a:$ & 4 & $0^{242}$ & 93 & $\begin{array}{l}24+4 \\
2\end{array}$ & $0^{12}$ & ${ }^{439}$ & $3^{3+4}$ & $\begin{array}{l}323 \\
2\end{array}$ & :364 & 630 & $i^{2,250}$ & $94+$ & 602 & 6 & $\begin{array}{l}120 \\
2\end{array}$ & \begin{tabular}{|l|}
423 \\
4
\end{tabular} & 420 & 1928 & $6^{103}$ & 2364 & $\mid$ & \\
\hline & & s & 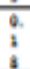 & 0,3 & $\because 4$ & $\because 4$ & ;" & \begin{tabular}{|l}
14. \\
24
\end{tabular} & 每. & $\because 2$ & 4 & is & 13 & $\begin{array}{l}70 \\
2\end{array}$ & 3 & 2.46 & 19. & 0.3 & 3 & 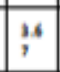 & 1.67 & 0.00 & $\because 4$ & ${ }_{2}^{24}$ & 0.44 & 0.30 & 3 & 2.32 & \\
\hline
\end{tabular}

Figure 14: 


\section{After trial (Figure 15)}

\begin{tabular}{|c|c|c|c|c|c|c|c|c|c|c|c|c|c|c|c|c|c|c|c|c|c|c|c|c|c|c|c|c|c|}
\hline \multirow[b]{3}{*}{ Sr.:Do } & \multirow[b]{3}{*}{ Defect Origis } & \multicolumn{28}{|c|}{ AFTER TRIAL DATA COLLECTION } \\
\hline & & \multirow[b]{2}{*}{ Name of Defect } & \multicolumn{26}{|c|}{ DAY } & \\
\hline & & & 1 & 2 & 3 & 4 & 5 & 6 & 7 & 8 & 9 & 10 & 11 & 12 & 13 & 14 & 15 & 16 & 17 & 18 & 19 & & 21 & 2 & 23 & 24 & 25 & Iotal pse & 4 \\
\hline \multirow{7}{*}{1} & \multirow{7}{*}{ Procesing } & Stain & 24 & 4 & & & & 43 & 15 & 45 & 40 & 40 & 6 & 5 & 3 & 28 & 17 & 8 & 12 & 246 & 16 & & 14 & 1 & 26 & 16 & & 609 & 135 \\
\hline & & Shearing & 20 & 18 & & & & & 1. & 7 & 25 & 7 & 6 & & & 1 & 1 & & 4 & 2 & & & 20 & & 1 & & & 109 & 25 \\
\hline & & Linting & 3 & & & & & & 1 & 23 & 54 & 49 & 43 & 13 & 28 & 12 & 4 & 17 & 8 & & & & 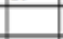 & & 15 & 11 & & 283 & 6.2 \\
\hline & & Bowing & & 1 & & & & & & & 1 & & & & & & & & 1 & & & & 1 & & & & & 3 & 0.06 \\
\hline & & Patby & & & & & & & & 4 & & & & & & & & & 2 & & 2 & & 2 & & & & & 10 & 0.2 \\
\hline & & Small hole & 2 & & 39 & 14 & 7 & 4 & 4 & 10 & 7 & 10 & 3 & & 1 & 6 & 2 & & & 15 & 38 & & 30 & 13 & & 2 & 14 & 250 & 55 \\
\hline & & Total & & & & & & & & & & & & & & & & & & & & & & & & & & 1310 & 0.37 \\
\hline \multirow{3}{*}{2} & \multirow{2}{*}{ Grey Folking } & Joint pikes & 4 & 14 & & & & & 4 & 3 & 1 & 6 & 14 & & 7 & & 6 & 18 & 7 & 2 & 21 & & 11 & 8 & 1 & 9 & & 136 & 3.02 \\
\hline & & Gry maxking & 7 & 11 & & & & 13 & 5 & 37 & 25 & 13 & 16 & 8 & 2 & 15 & 11 & 15 & 3 & 8 & $5 !$ & & 18 & 8 & & 2 & & 268 & 59 \\
\hline & & Total & & & & & & & & & & & & & & & & & & & & & & & & & & 404 & 0.11 \\
\hline \multirow{8}{*}{3} & \multirow{7}{*}{ Fisih Fol6ang } & Legth est & 13 & 4 & 24 & & & 24 & 16 & 23 & 10 & 14 & 16 & 4 & & 2 & 6 & 6 & 2 & 5 & 39 & & 30 & 15 & 7 & 5 & & 267 & 59 \\
\hline & & \begin{tabular}{|l|} 
Crom ent \\
\end{tabular} & 14 & & 7 & & & & 2 & 12 & 5 & 1 & 1. & 1 & & + & 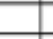 & 26 & 3 & 38 & 48 & $58]$ & 11 & 3 & & 6 & 15 & 251 & 5.5 \\
\hline & & Setvedge cet & 4 & 4 & 27 & 33 & 23 & & 7 & 2 & + & 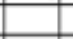 & & 5 & & 2 & + & 1 & 4 & 4 & 13 & 652 & 22 & & 2 & & 81 & 284 & 63 \\
\hline & & Crom stich $0 \mathrm{~T}$ & 2 & 8 & 23 & 3 & & 33 & 66 & 48 & 29 & 28 & & 26 & 9 & 18 & 19 & 76 & 14 & 499 & 56 & & 13 & 13 & 2 & 16 & & 1021 & 22.7 \\
\hline & & \begin{tabular}{|l|} 
Legth stich $0 \mathrm{~T}$ \\
\end{tabular} & & 24 & & & & 5 & 20 & 8 & 1 & 4 & 25 & 3 & 1 & 1 & 5 & 5 & & 13 & 32 & & 6 & 1 & 6 & & & 160 & 35 \\
\hline & & Hole stiching & & & & & & & & & & & & & & & & & & & & & 22 & & & & 12 & 41 & 09 \\
\hline & & Boeds cut & & & 3 & 1 & & & 1 & & & & & & & & & & & & 3 & & & & & & & 8 & 0.17 \\
\hline & & Total & & & & & & & & & & & & & & & & & & & & & & & & & & 2088 & 0.6 \\
\hline \multirow{7}{*}{4} & \multirow{7}{*}{ Weaving } & \begin{tabular}{|l|} 
Marieg end \\
\end{tabular} & & 3 & & & & 10 & 13 & 20 & 4 & 22. & 39 & 15 & 6 & 3 & 7 & 2 & 3 & 36 & 10 & & 8 & & 15 & 4 & & 221 & 4.1 \\
\hline & & Pile gutling & 3 & 2 & & & & 2 & 3 & 2 & 16 & 12 & 17 & 4 & 3 & 1 & & 2 & & 6 & 12 & & 15 & 12 & 2 & & & 114 & 2.5 \\
\hline & & Cracs & 1 & 2 & & & & 13 & & 8 & 8 & 49 & 22 & 21. & 26 & 4 & 9 & 5 & & 41 & & & & & 4 & 2 & & 215 & 53 \\
\hline & & Half piecer & & & 23 & & 23 & & & & & & & & & & & & & & & 7 & + & & & & & 53 & 11 \\
\hline & & \begin{tabular}{|l|} 
Short pitces \\
\end{tabular} & & & & 34 & 11 & & & & & & & & & & & & & & & & & & & & & 45 & 1 \\
\hline & & Reverse Terry & & & & & & & & & & 3 & & & & & & & & & 7 & & 3 & 2 & & & & 15 & 0.3 \\
\hline & & Lining & & & & & & & & & & & & & & & & & & & 2 & & & & 5 & & & 7 & 0.1 \\
\hline & & Iotal & & & & & & & & & & & & & & & & & & & & & & & & & & 670 & 0.1 \\
\hline & & Iotal & 100 & 95 & 153 & 85 & 80 & 167 & 173 & 252 & 226 & 2585 & & 105 & 86 & 93 & 87. & 181 & 34 & 911 & 370 & 175 & 229 & 76 & 86 & 73 & 157 & 4276 & 1.2 \\
\hline & & CTN packing ( $\mathrm{Pe}$ & 25128 & 9706 & 10292 & 14760 & 15480 & 14832 & 15030 & 13560 & 15894 & 189801 & $15842]$ & 17656 & \begin{tabular}{l|l|l}
3552 & 40 \\
\end{tabular} & $\begin{array}{lll}40930 & 9\end{array}$ & 9406 & 26556 [ & 19068 & 12972 & 8676 . & 19146 ] & 10818 & 12026 & 4658 & 12937 & 17545 & \begin{tabular}{|l|}
347620 \\
\end{tabular} & \\
\hline
\end{tabular}

Figure 15:

Result and Discussion

Percent defects in all departments (Figure 16,17)

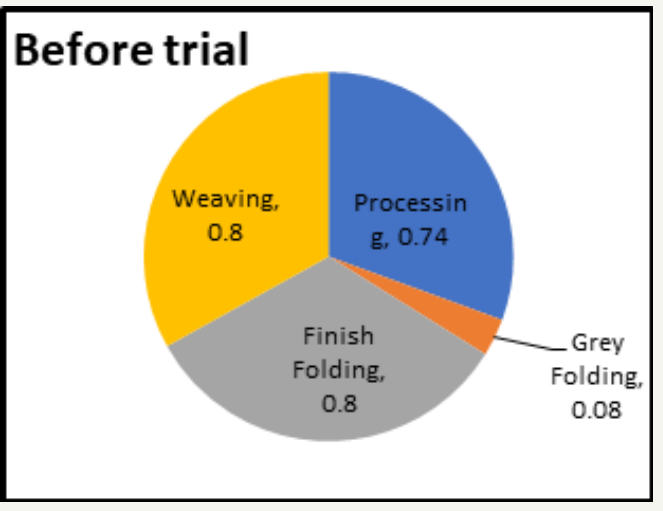

Figure 16: Defect of percentage in all departments.

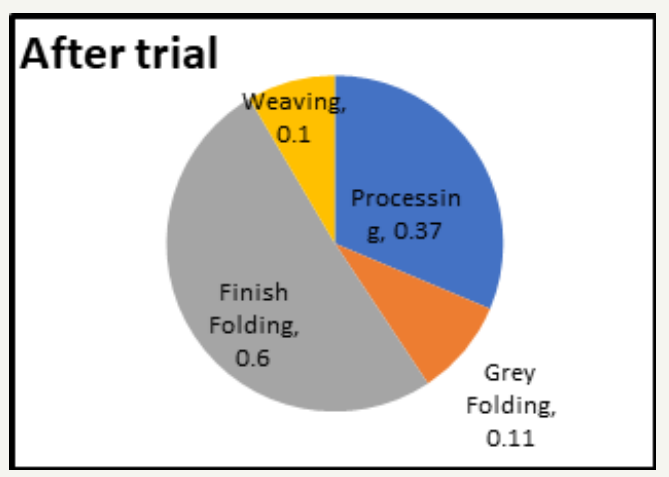

Figure 17: Defect of percentage in all departments. 
Percentage of processing defect before trial (25 Days) (Table 3)

Table 3:

\begin{tabular}{|c|c|c|c|}
\hline Sr. No. & Name of Defect & Total Defect & Defect \% \\
\hline 1 & Stain & 823 & 19.7 \\
\hline 2 & Shearing & 190 & 4.5 \\
\hline 3 & Lintting & 83 & 1.9 \\
\hline 4 & Bowing & 21 & 0.5 \\
\hline 5 & Patchy & 13 & 0.3 \\
\hline 6 & Hole & 300 & 7.2 \\
\hline 7 & Big hole & 27 & 0.6 \\
\hline \multicolumn{2}{|c|}{ Total defect } & 1457 & 0.74 \\
\hline \multicolumn{2}{|c|}{ CTN Packing (Pcs) } & \multicolumn{2}{|c|}{195635} \\
\hline
\end{tabular}

\section{Graphical representation}

Before trial result of defect in processing department (Figure 18):

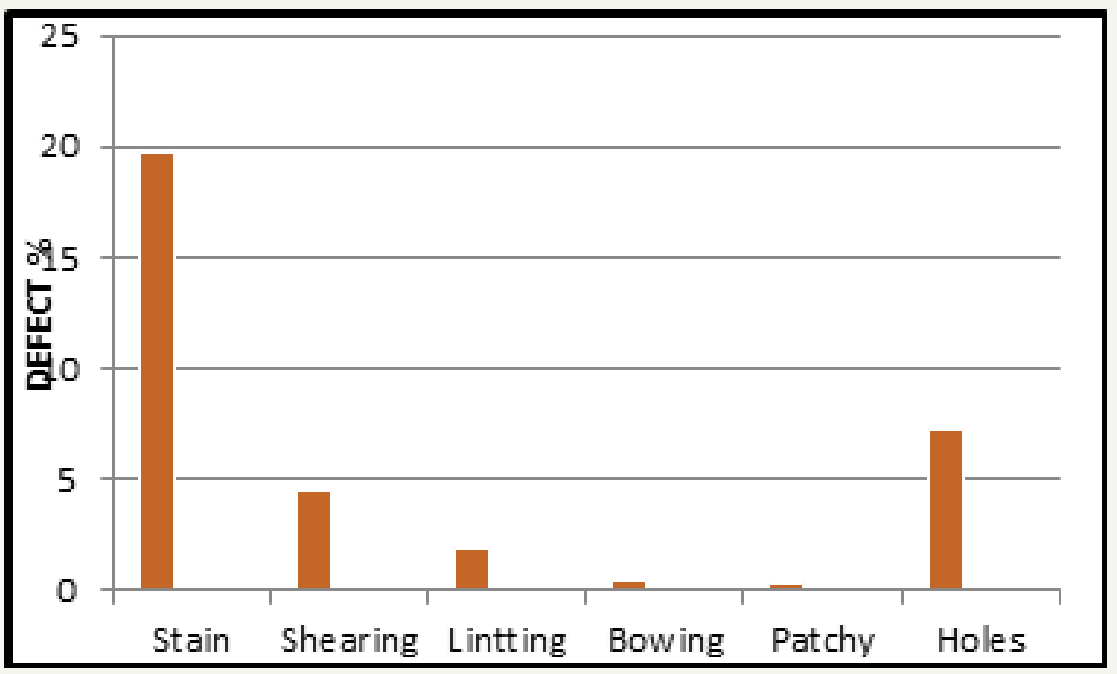

Figure 18:

Percentage of processing defect after trial (25 Days) (Table 4):

Table 4:

\begin{tabular}{|c|c|c|c|}
\hline Sr. No. & Name of Defect & Total Defect & Defect \% \\
\hline 1 & Stain & 609 & 13.5 \\
\hline 2 & Shearing & 109 & 2.5 \\
\hline 3 & Lintting & 283 & 6.2 \\
\hline 4 & Bowing & 3 & 0.06 \\
\hline 5 & Patchy & 10 & 0.2 \\
\hline 6 & Hole & 250 & 5.5 \\
\hline 7 & Big hole & 46 & 1.03 \\
\hline \multicolumn{2}{|c|}{ Total defect } & 1310 & 0.37 \\
\hline \multicolumn{2}{|c|}{ CTN Packing (Pcs) } & \multicolumn{2}{|c|}{347620} \\
\hline
\end{tabular}




\section{Graphical representation}

After trial result defect in processing department (Figure 19):

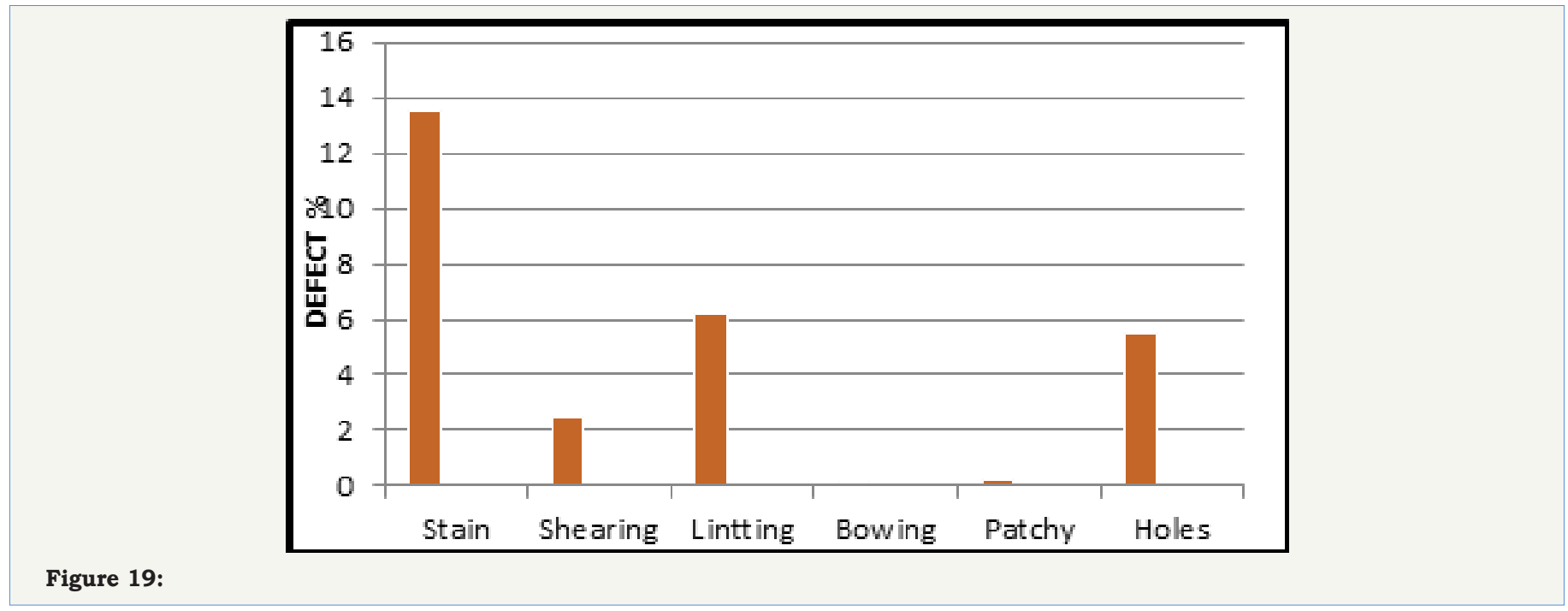

Defect in processing before trial and after trial (Table 5 \&Figure 20):

Figure 20:

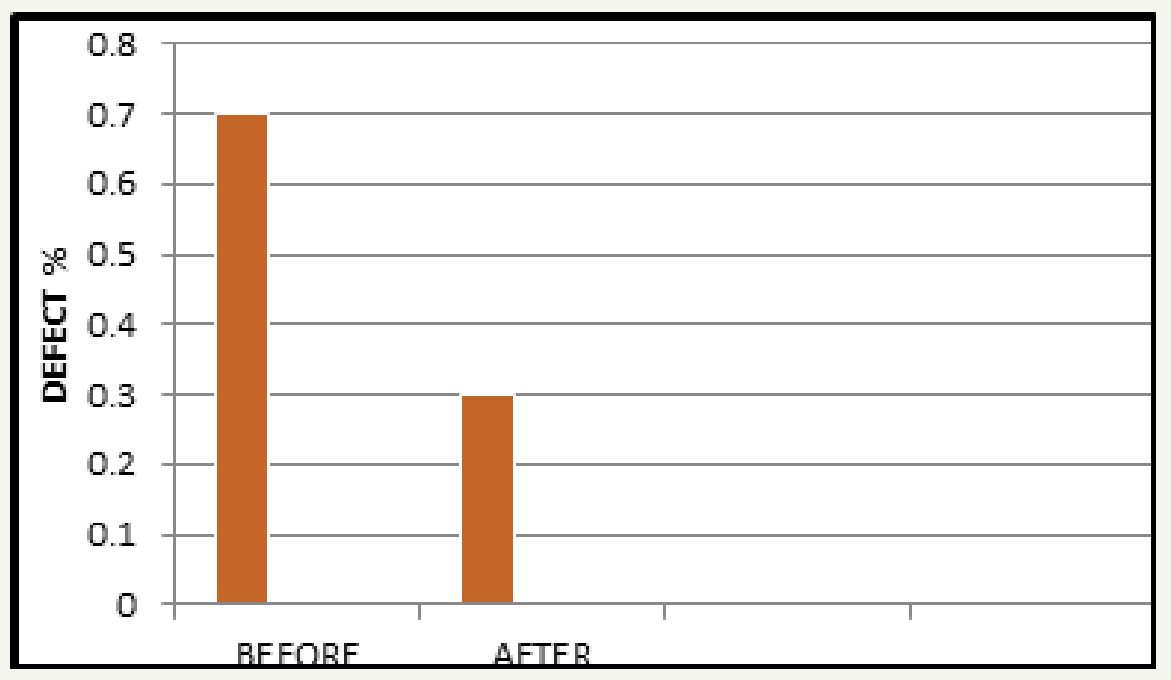

Table 5:

\begin{tabular}{|c|c|}
\hline \multicolumn{2}{|c|}{ Percent of Defect } \\
\hline Before trial & After trial \\
\hline $0.74 \%$ & $0.37 \%$ \\
\hline
\end{tabular}

\section{Discussion}

In this project we try to minimize the dyeing defect arising in the processing department. For this work first we study and analyze that which defects are occurs in terry towel production and their quantity by collecting back month data of downgrade in overall terry towel production [5]. After this we calculate \% wise defectives in various department.

Then we focus on defect arising in processing department. By our above analysis processing defect was found in $0.7 \%$ of total CTN packing (Pcs) in which stain was found in large quantity $19.7 \%$, Shearing $4.5 \%$, Lintting $1.9 \%$ [6], Bowing 0.5\%, uneven dyeing(patchy) $0.3 \%$ and holes found $7.2 \%$ Near by one month we are continue this work and then after discussion with our industrial mentor we try to goes towards the origin of defect and suggest the remedies for defect occurs in processing department [7,8]. After taking corrective action by implementation of our remedies we start work for minimization of defect near by one month. After completion of work we analyze again quantity of defect in processing department which is slightly minimize than before trial defect percent in which stain was found $13.5 \%$, shearing $2.5 \%$, bowing $0.06 \%$, patchy dyeing $0.2 \%$ and holes $5.5 \%$. After comparing it with previous data of defect our project work minimizes the processing defect from $0.7 \%$ to $0.3 \%$ i.e we minimize $0.4 \%$ defect in processing department $[9,10]$.

\section{Conclusion}

In this project from our above analysis the defect coming from processing department was minimize as follow: 
i. In conventional process of industry stains was coming $19.7 \%$ and after our project work and implementation of our suggested remedies it is minimize to $13.5 \%$.

ii. Shearing was coming $4.5 \%$ and after implementation of remedies it is minimize to $2.5 \%$.

iii. Bowing was coming $0.5 \%$ and after implementation remedies it is minimized to $0.06 \%$.

iv. Uneven dyeing (patchy) was coming $0.3 \%$ and after implementation of remedies it is minimize to $0.2 \%$.

v. In conventional industrial process of industry, a hole is coming $7.2 \%$ and after our studies and action taken it is minimized to $5.5 \%$.

From our above report we concluded that before taking corrective action overall percentage of processing defect was $0.7 \%$. After taking corrective action in processing department by implementation of our remedies, defect coming from over all processing department was $0.3 \%$. Defect arising in processing department were under controlled and minimizing by $0.4 \%$ differences. In this project it was also concluded that most of the processing defect were coming due to manual operation during unloading and loading processing.

\section{References}

1. Islam, Moyinul M, Sakil Mahmud (2015) Study on different types of dyeing faults, causes and remedies of knit fabrics. Int J Eng-Technol 15(2): 6-15.

2. Patil K (2014) Colour bleeding problems in cotton yarn dyed fabrics dyed with reactive colours \& some remedial measures, pp: 20-25.

3. Subhash JP. Manufacturing of terry towel.

4. Broadbent AD (2001) Basic principles of textile coloration, pp: 332-357.

5. Chinta PS, Dhar S (2007) Problems in dyeing and their remedies.

6. Savita P, Tushar AS, Madhuri K, Sujit G, Raichurkar PP. Lab to bulk shade variation in terry towel fabric for RFT dyeing. International dyer \& Textile Finisher 4(4): 40-43.

7. Tomasino C (1992) Chemistry \& technology of fabric preparation \& finishing. North Carolina State University, North Carolina, USA.

8. Perkins, Warren S (1996) Textile coloration and finishing. Carolina Academic Press, Durham, North Carolina, USA.

9. Amin MT (2014) Overview of a knit-dyeing factory with necessary production formulas. Global Journal of Research in Engineering 14(1): $1-7$.

10. Moin CJ, Mahabubuzzaman AKM (2009) Process for level dyeing of $100 \%$ cotton knit fabrics with reactive dye. J Innov Dev Strategy 3 (4): $1-8$
Creative Commons Attribution 4.0 International License

For possible submissions Click Here

\section{Submit Article}

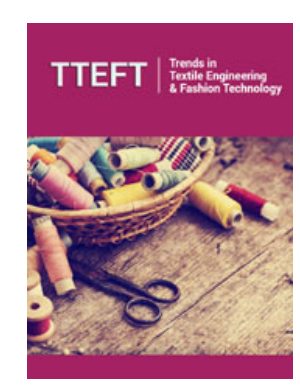

Trends in Textile Engineering \& Fashion Technology

\section{Benefits of Publishing with us}

- High-level peer review and editorial services

- Freely accessible online immediately upon publication

- Authors retain the copyright to their work

- Licensing it under a Creative Commons license

- Visibility through different online platforms 